\title{
Classification of "Pseudomonas azotocolligans" Anderson 1955, 132, in the Genus Sphingomonas as Sphingomonas trueperi sp. nov.

\author{
PETER KÄMPFER, ${ }^{1}$ EWALD B. M. DENNER, ${ }^{2}$ SVENJA MEYER, ${ }^{1}$ EDWARD R. B. MOORE, ${ }^{3}$ \\ AND HANS-JÜRGEN BUSSE ${ }^{2 *}$ \\ Institut für Angewandte Mikrobiologie, Justus-Liebig-Universität Giessen, D-35390 Giessen, ${ }^{1}$ and Bereich \\ Mikrobiologie, Gesellschaft für Biotechnologische Forschung, D-38124 Braunschweig, ${ }^{3}$ Germany, \\ and Institut für Mikrobiologie und Genetik, Universität Wien, A-1030 Vienna, Austria ${ }^{2}$
}

\begin{abstract}
"Pseudomonas azotocolligans" ATCC $12417^{\mathrm{T}}$ ( $\mathrm{T}=$ type strain), which was described as a diazotrophic bacterium, was reinvestigated to clarify its taxonomic position. $16 \mathrm{~S}$ ribosomal DNA sequence comparisons demonstrated that this strain clusters phylogenetically with species of the genus Sphingomonas and represents a new species. The results of investigations of the fatty acid patterns, polar lipid profiles, and quinone system supported this placement. The substrate utilization profile and biochemical characteristics displayed no obvious similarity to the characteristics of any previously described species of the genus Sphingomonas. The new name Sphingomonas trueperi is proposed on the basis of these results and previously published data for the $G+C$ content of the genomic DNA and the polyamine pattern.
\end{abstract}

In a study of nitrogen-fixing soil bacteria, Anderson (1a) described a diazotrophic, polarly flagellated, rod-shaped organism and proposed the name "Pseudomonas azotocolligans" for it. Only one strain was placed in " $P$. azotocolligans," and this strain was deposited in the National Collection of Industrial Bacteria as strain NCIB $9391^{\mathrm{T}}(\mathrm{T}=$ type strain). However, the name " $P$. azotocolligans" has not been validated. The $\mathrm{G}+\mathrm{C}$ content of the DNA of this organism was determined to be $65.6 \mathrm{~mol} \%$ (5), and, based on the detection of peritrichous flagella, De Ley (5) proposed that "P. azotocolligans" should be removed from the genus Pseudomonas. Subsequently, it was shown that this organism could not fix nitrogen (8). In the American Type Culture Collection catalog (1), strain NCIB $9391^{\mathrm{T}}$ is listed as a patent strain for the production of coenzyme Q-10. The major component in the polyamine pattern has been demonstrated to be sym-homospermidine (4). However, neither ubiquinone 10 nor sym-homospermidine is found in the species belonging to the genus Pseudomonas sensu stricto $(2,4,17)$, as defined phylogenetically $(6,24)$. The results of DNA-rRNA hybridization studies distinguished " $P$. azotocolligans" from the authentic Pseudomonas species and placed it close to the misnamed organisms Pseudomonas paucimobilis and Flavobacterium capsulatum phylogenetically $(3,7)$. Recently, the latter two species have been reclassified as species of the genus Sphingomonas (25), which now includes the following validly described species: Sphingomonas paucimobilis (type species of the genus), Sphingomonas adhaesiva, Sphingomonas asaccharolytica, Sphingomonas capsulata, Sphingomonas chlorophenolica, Sphingomonas macrogoltabidus, Sphingomonas mali, Sphingomonas parapaucimobilis, Sphingomonas pruni, Sphingomonas rosa, Sphingomonas sanguis, Sphingomonas terrae, and Sphingomonas yanoikuyae $(13,15,16,21,22,25)$. Phylogenetically, the species of the genus Sphingomonas form a rather homogeneous cluster that is also supported by several common chemotaxonomic features. Sphingomonads are characterized by the presence of ubiquinone 10 , the presence of a unique sphingoglycolipid, the presence of 2-hydroxy fatty acids, the

\footnotetext{
* Corresponding author. Mailing address: Institut für Mikrobiologie und Genetik, Universität Wien, Dr. Bohr-Gasse 9, A-1030 Vienna, Austria. Phone: 43-1-79515-4121. Fax: 43-1-79515-4114. E-mail: elliot @gem.univie.ac.at.
}

absence of 3-hydroxy fatty acids, and the presence of spermidine or sym-homospermidine as the major polyamine $(4,16$, $17,22,25)$.

Only one strain of "P. azotocolligans" is available in culture collections. We reinvestigated the taxonomic position of " $P$. azotocolligans" ATCC $12417^{\mathrm{T}}\left(=\mathrm{NCIMB} 9391^{\mathrm{T}}=\mathrm{LMG} 2142^{\mathrm{T}}=\right.$ DSM $7225^{\mathrm{T}}$ ), which was obtained from G. Auling (Institute of Microbiology, University of Hannover, Hannover, Germany). " $P$. azotocolligans" was characterized by analyzing the quinone system and the polar lipids by thin-layer chromatography $(14$, 23 ), by performing a gas-liquid chromatographic analysis of the fatty acids (10), by determining physiological and biochemical characteristics $(9,18)$, and by determining the $16 \mathrm{~S}$ rRNA gene sequence (15) and was compared to the previously established species of the genus Sphingomonas.

" $P$. azotocolligans" contained a ubiquinone with 10 isoprenoid units in the side chain (ubiquinone Q-10) and a sphingoglycolipid. The overall polar lipid pattern of this strain was similar to the patterns determined for the other Sphingomonas species in that it includes phosphatidylethanolamine, phosphatidylglycerol, and diphosphatidylglycerol as the major compounds (Fig. 1). Other lipids detected in " $P$. azotocolligans" were high levels of phosphatidyldimethylethanolamine and an unidentified lipid and minor levels of phosphatidylcholine, phosphatidylmonomethylamine, an unidentified glycolipid (GL2), an unidentified lipid, and two unidentified phospholipids (PL1 and PL3). This polar lipid profile clearly distinguished " $P$. azotocolligans" from the other Sphingomonas species (Table 1).

Fatty acid methyl esters were extracted and prepared by the standard protocol of the Microbial Identification System (MIDI; Microbial ID, Inc., Newark, Del.). Extracts were analyzed by using a Hewlett-Packard model HP6890A gas chromatograph equipped with a flame ionization detector, an automatic sampler, an integrator, and a computer, as described previously (11). All Sphingomonas strains contained the fatty acids $16: 0$, summed feature $7(18: 1 \omega 7 \mathrm{c}, 18: 1 \omega 9 \mathrm{t}$, and/or $18: 1 \omega 12 \mathrm{t})$, and 14:0 $2 \mathrm{OH}$, and all but four strains contained summed feature 4 (16:1 $\omega 7 \mathrm{c}$ and/or 15:0 iso $2 \mathrm{OH}$ ) (Table 2). These fatty acids have also been detected in previous studies $(21,22,25)$ and are characteristic of all validly described species of the genus Sphingomonas. A more precise comparison of the results of this study with the results described by Yabuuchi et al. (25) and Takeuchi et al. $(21,22)$ was not possible due to the different 


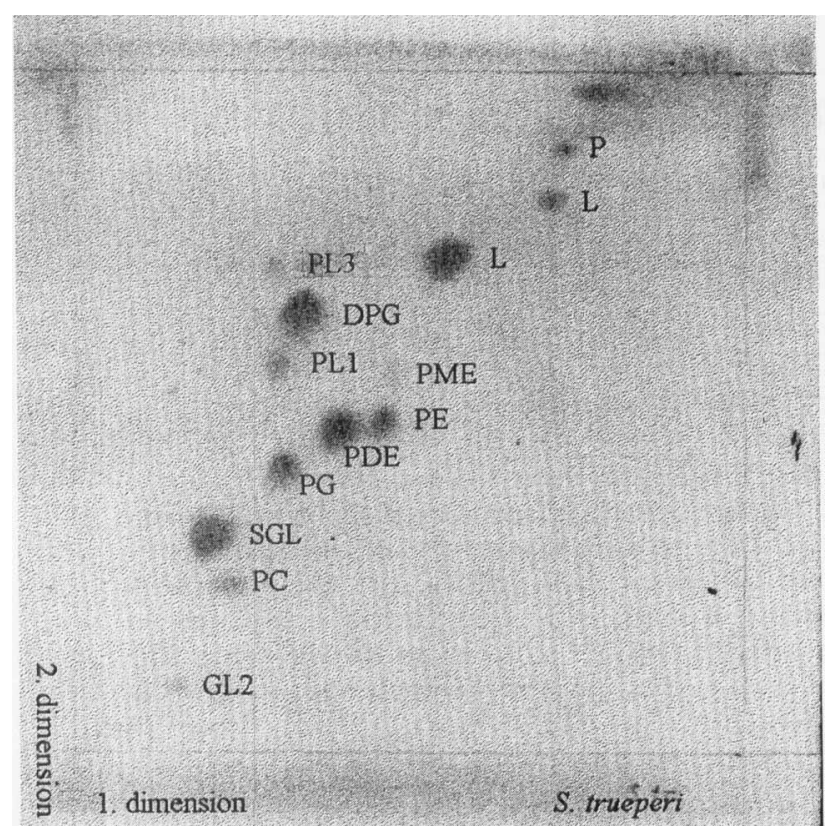

FIG. 1. Polar lipids of $S$. trueperi DSM $7225^{\mathrm{T}}$ after separation by two-dimensional thin-layer chromatography. Abbreviations: $\mathrm{PE}$, phosphatidylethanolamine; PME, phosphatidylmonomethylethanolamine; PG, phosphatidylglycerol; DPG, diphosphatidylglycerol; PDE, phosphatidyldimethylethanolamine; PC, phosphatidylcholine; SGL, sphingoglycolipid; PL1 and PL3, unidentified phospholipids; GL2, unidentified glycolipid; L, unidentified polar lipids; P, pigment.

methods of fatty acid extraction and analysis used. Table 2 shows that the type strains of Sphingomonas species exhibit considerable heterogeneity in their fatty acid profiles, which correlates with the heterogeneity found in the polar lipid profiles. The presence of 2-hydroxymyristic acid $(14: 02 \mathrm{OH})$ as the dominant hydroxylated fatty acid $(0.8$ to $18.8 \%)$ and the absence of 3-hydroxylated fatty acids are consistent with and confirm the results of other studies. However, the relatively high percentage of 2-hydroxymyristic acid described in previous reports $(21,22)$ could not be confirmed. $S$. terrae IFO $15098^{\mathrm{T}}$ was observed to contain 15:0 2OH as a second hydroxylated fatty acid, which could not be detected in the type strain of any other species. This finding has been described previously by Takeuchi et al. (21). In contrast to the results of Takeuchi et al. (22), no 15:0 $2 \mathrm{OH}$ was detected in the type strains of $S$. pruni, $S$. asaccharolytica, and $S$. mali, whereas $16: 02 \mathrm{OH}$ was detected in $S$. macrogoltabidus (in agreement with the results of Takeuchi et al. [21]) and $S$. yanoikuyae (in agreement with the results of Takeuchi et al. [22]) but not in $S$. mali and $S$. pruni (in contrast to the findings of Takeuchi et al. [22]).

Furthermore, all strains were characterized on the basis of 87 biochemical and physiological characteristics as described previously (12), and the results are shown in Table 3 . Where possible, a detailed comparison was made with all previously published data $(21,22,25)$. Table 3 shows that nearly all test results which could be compared directly with data from previously published papers were confirmed. However, it should be noted that acid formation from various sugars often resulted in very weak positive results; these test results should be interpreted with care. Table 3 shows that the species described in this paper, Sphingomonas trueperi, can be differentiated from all other Sphingomonas species on the basis of the results of several tests. Additional biochemical characteristics of $S$. trueperi are given below. A numerical analysis of the data was performed by using the simple matching coefficient and the Jaccard coefficient $\left(S_{\mathrm{J}}\right)(20)$, and phenograms were generated by using the unweighted pair group with mathematical average (UPGMA) algorithm. Similar phenograms were generated with the two coefficients. The results of the UPGMA-S J $_{\mathbf{J}}$ analysis are shown in Fig. 2.

A nearly complete $16 \mathrm{~S}$ rRNA gene sequence (nucleotide positions 28 to 1524; Escherichia coli numbering) of " $P$. azotocolligans," comprising 1,442 nucleotide positions, was determined by PCR amplification and direct sequencing of the PCR-amplified 16S ribosomal DNA (rDNA). A comparative analysis and estimation of phylogenetic relationships demonstrated that " $P$. azotocolligans" clusters within the genus Sphingomonas and is most closely related to $S$. paucimobilis, $S$. parapaucimobilis, $S$. sanguis, and $S$. adhaesiva (Fig. 3). However, a comparison of levels of $16 \mathrm{~S}$ rDNA sequence similarity (Table 4) revealed that " $P$. azotocolligans" represents a distinct species of the genus Sphingomonas. The levels of sequence similarity between the 16S rRNA genes of "P. azotocolligans" and other Sphingomonas species ranged from $95.8 \%$ (with the sequence of $S$. paucimobilis and with the nearly complete sequences of $S$.

TABLE 1. Polar lipid distribution in Sphingomonas species

\begin{tabular}{|c|c|c|c|c|c|c|c|c|c|c|c|c|c|c|c|c|c|}
\hline \multirow{2}{*}{ Organism } & \multicolumn{17}{|c|}{ Distribution of the following polar lipid ${ }^{a}$} \\
\hline & PME & PE & PG & DPG & PDE & $\mathrm{PC}$ & SGL & APL1 & PL1 & PL2 & PL3 & GL1 & GL2 & GL3 & GL4 & L1 & $\mathrm{L} 2$ \\
\hline S. trueperi DSM $7225^{\mathrm{T}}$ & $\square$ & $\mathbf{\square}$ & & $\mathbf{\square}$ & $\mathbf{\square}$ & $\square$ & D & - & $\square$ & - & $\square$ & - & $\square$ & - & - & $\mathbf{\square}$ & $\square$ \\
\hline S. mali IFO $15500^{\mathrm{T}}$ & $\square$ & $\square$ & & $\mathbf{\square}$ & $\boldsymbol{\square}$ & $\square$ & $\mathbf{\square}$ & - & $\square$ & - & $\square$ & - & $\square$ & $\square$ & - & $\mathbf{\square}$ & $\square$ \\
\hline S. pruni IFO $15498^{\mathrm{T}}$ & $\square$ & $\square$ & & $\mathbf{\square}$ & $\mathbf{\square}$ & $\square$ & $\mathbf{\square}$ & - & $\square$ & - & $\square$ & - & $\square$ & $\square$ & - & $\mathbf{\square}$ & $\square$ \\
\hline S. asaccharolytica IFO $15499^{\mathrm{T}}$ & $\square$ & $\square$ & $\overline{\mathbf{a}}$ & $\overline{\mathbf{a}}$ & $\overline{\mathbf{n}}$ & $\square$ & $\overline{\mathbf{Z}}$ & - & $\square$ & - & $\bar{\square}$ & - & $\square$ & $\square$ & - & $\overline{\mathbf{\square}}$ & $\square$ \\
\hline S. paucimobilis DSM $1098^{\mathrm{T}}$ & - & $\mathbf{\square}$ & 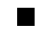 & $\square$ & - & $\boldsymbol{\square}$ & $\mathbf{\square}$ & - & - & - & $\square$ & - & $\square$ & - & - & - & - \\
\hline S. parapaucimobilis IFO $15100^{\mathrm{T}}$ & $\square$ & & & $\bar{\square}$ & $\square$ & $\overline{\mathbf{u}}$ & $\square$ & - & - & $\square$ & $\square$ & $\mathbf{a}$ & $\square$ & - & - & - & - \\
\hline S. adhaesiva IFO $15099^{\mathrm{T}}$ & $\square$ & a & & 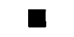 & $\square$ & $\mathbf{0}$ & $\bar{\square}$ & $\square$ & - & $\overline{\mathbf{\square}}$ & $\square$ & - & $\square$ & - & - & - & - \\
\hline S. sanguis IFO $13937^{\mathrm{T}}$ & - & $\mathbf{\square}$ & & $\mathbf{\square}$ & $\square$ & $\mathbf{m}$ & $\square$ & - & - & - & $\square$ & $\square$ & $\square$ & - & - & - & - \\
\hline S. yanoikuyae IFO $15102^{\mathrm{T}}$ & $\square$ & $\bar{\square}$ & & $\overline{\mathbf{\square}}$ & $\square$ & $\vec{\square}$ & $\boldsymbol{\square}$ & $\square$ & $\square$ & - & - & $\mathbf{\square}$ & - & - & - & - & - \\
\hline S. chlorophenolica ATCC $33790^{\mathrm{T}}$ & $\mathbf{a}$ & $\mathbf{\square}$ & & $\mathbf{\square}$ & $\mathbf{\square}$ & $\square$ & $\mathbf{0}$ & - & - & $\square$ & $\square$ & - & - & - & - & - & - \\
\hline S. macrogoltabidus IFO $15033^{\mathrm{T}}$ & $\square$ & & & & - & $\square$ & $\square$ & - & - & - & $\square$ & $\square$ & {$[\square$} & - & $\square$ & - & - \\
\hline S. terrae IFO $15090^{\mathrm{T}}$ & $\square$ & $\bar{\square}$ & & 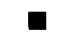 & $\square$ & $\mathbf{\square}$ & $\mathbf{\square}$ & - & - & - & $\square$ & $\square$ & - & - & - & - & - \\
\hline S. rosa IFO $15208^{\mathrm{T}}$ & $\square$ & $\mathbf{\square}$ & & & $\square$ & $\square$ & $\mathbf{\square}$ & $\square$ & - & $\square$ & $\square$ & - & $\square$ & - & - & - & - \\
\hline S. capsulata DSM $30196^{\mathrm{T}}$ & $\square$ & $\mathbf{\square}$ & $\mathbf{\square}$ & $\mathbf{\square}$ & $\mathbf{a}$ & $\square$ & $\mathbf{\square}$ & $\square$ & - & $\square$ & $\square$ & - & $\square$ & - & - & - & - \\
\hline
\end{tabular}

a PME, phosphatidylmonomethylethanolamine; PE, phosphatidylethanolamine; PG, phosphatidylglycerol; DPG, diphosphatidylglycerol, PDE, phosphatidyldimeth ylethanolamine; PC, phosphatidylcholine; SGL, sphingoglycolipid; APL1, unidentified aminophospholipid; PL1, PL2, and PL3, unidentified phospholipids; GL1, GL2, GL3, and GL4, unidentified glycolipids; L1 and L2, unidentified lipids. $\mathbf{\square}$, present in major amounts; $\square$, present in minor amounts; - , not detected. 
TABLE 2. Major fatty acid compositions of the type strains of 14 Sphingomonas species ${ }^{a}$

\begin{tabular}{|c|c|c|c|c|c|c|c|c|c|c|c|c|c|c|}
\hline \multirow[b]{2}{*}{ Compound $^{b}$} & \multicolumn{14}{|c|}{$\%$ in: } \\
\hline & 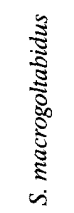 & 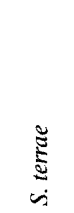 & 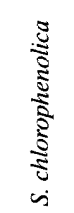 & 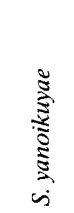 & 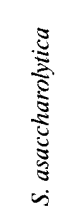 & 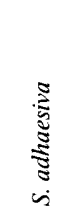 & 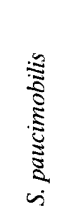 & 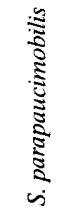 & 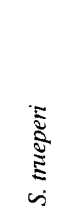 & 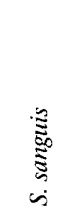 & 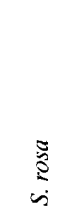 & 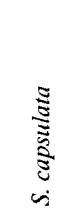 & $\begin{array}{l}\overline{\bar{\Xi}} \\
\dot{\tilde{s}}\end{array}$ & $\begin{array}{l}\overline{\mathbf{z}} \\
\dot{5} \\
\dot{s}\end{array}$ \\
\hline \multicolumn{15}{|l|}{ Saturated fatty acids } \\
\hline $14: 0$ & & & & 0.5 & & & 1.4 & 1.0 & & 1.0 & 1.5 & & & \\
\hline $15: 0$ & & 5.9 & & & & & & & & 1.1 & & & & \\
\hline $16: 0$ & 12.8 & 4.5 & 9.5 & 11.2 & 13.3 & 6.5 & 8.7 & 9.6 & 9.8 & 13.6 & 10.9 & 8.1 & 11.8 & 16.0 \\
\hline $17: 0$ & & 3.8 & & & & & & & 1.6 & 0.5 & & & & \\
\hline $18: 0$ & & & & & & & & & & 0.6 & & & & \\
\hline \multicolumn{15}{|l|}{ Unsaturated fatty acids } \\
\hline $16: 1 \omega 5 \mathrm{c}$ & 2.9 & 2.1 & 2.4 & 2.7 & & & & 1.9 & & 1.0 & & 1.7 & & \\
\hline $17: 1 \omega 6 c$ & 4.5 & 48.1 & 6.4 & 3.2 & & 21.2 & 3.0 & 3.8 & 13.6 & 2.4 & & 4.0 & 9.3 & \\
\hline $17: 1 \omega 8 \mathrm{c}$ & & 7.9 & & & & & & & & & & & & \\
\hline $18: 1 \omega 5 \mathrm{c}$ & 0.9 & 0.6 & 2.5 & 2.1 & & & 3.2 & 3.5 & 4.0 & 3.5 & & & & \\
\hline \multicolumn{15}{|l|}{ Hydroxy fatty acids } \\
\hline $14: 02 \mathrm{OH}$ & 3.0 & 0.8 & 9.4 & 7.1 & 18.8 & 13.8 & 6.4 & 6.7 & 6.7 & 5.0 & 12.8 & 14.7 & 8.4 & 10.9 \\
\hline $15: 02 \mathrm{OH}$ & & 4.9 & & & & & & & & & & & & \\
\hline $16: 02 \mathrm{OH}$ & 2.9 & & & 0.9 & & & & & & & & & & \\
\hline \multicolumn{15}{|l|}{ Summed features } \\
\hline Summed feature 4 & 31.8 & 8.0 & 9.6 & 16.3 & & 6.2 & 2.7 & 8.6 & & 6.7 & 22.8 & 6.7 & & \\
\hline Summed feature 7 & 41.2 & 13.4 & 60.1 & 56.0 & 67.8 & 47.3 & 74.6 & 65.0 & 64.2 & 64.6 & 52.0 & 64.8 & 70.5 & 73.1 \\
\hline
\end{tabular}

${ }^{a}$ All strains except $S$. pruni IFO $15498^{\mathrm{T}}$ and $S$. mali IFO $15500^{\mathrm{T}}$ were grown on Trypticase soy broth agar at $28^{\circ} \mathrm{C}$ for $48 \mathrm{~h}$ prior to fatty acid analysis. As $S$. pruni IFO $15498^{\mathrm{T}}$ and $S$. mali IFO $15500^{\mathrm{T}}$ did not grown on Trypticase soy broth agar, they were cultured on Czapek Dox agar for $48 \mathrm{~h}$ at $28^{\circ} \mathrm{C}$

${ }^{b}$ For unsaturated fatty acids the position of the double bond is located by counting from the methyl ( $\left.\omega\right)$ end of the carbon chain. cis is indicated by the suffix $c$. Summed features are groups of two or three fatty acids that cannot be separated by gas-liquid chromatography with the MIDI system. Summed feature 4 contained one or more of the following fatty acids: $16: 1 \omega 7 \mathrm{c}$ and $15: 0$ iso $2 \mathrm{OH}$. Summed feature 7 contained one or more of the following isomers: $18: 1 \omega 7 \mathrm{c}, 18: 1 \omega 9 \mathrm{t}$, and $18: 1$ $\omega 12 \mathrm{t}$ (trans isomers are indicated by the suffix $\mathrm{t}$ ).

mali, S. pruni, and S. asaccharolytica, which were determined in this study) to $90.8 \%$ (with the sequence of $S$. capsulata). These data demonstrate that " $P$. azotocolligans," as well as $S$. mali, $S$. pruni, and $S$. asaccharolytica, are located on the $S$. paucimobilis phylogenetic branch (Fig. 3). "P. azotocolligans" and other species belonging to the $S$. paucimobilis branch (Fig. 3), including $S$. pruni and $S$. mali, have been shown to contain symhomospermidine as their major polyamine $(4,18,22)$. In contrast, $S$. asaccharolytica has been described as containing spermidine as the predominant compound (22). A reinvestigation of the polyamine pattern of $S$. asaccharolytica, performed under standardized conditions as described previously (4), revealed that this species also contained the major compound sym-homospermidine $(56.2 \mu \mathrm{mol} / \mathrm{g}$ [dry weight]), whereas spermidine $(3.6 \mu \mathrm{mol} / \mathrm{g}$ [dry weight]) and spermine $(1.2 \mu \mathrm{mol} / \mathrm{g}$ [dry weight]) were present only in minor amounts. As other branches in the genus, represented by $S$. capsulata, $S$. terrae, and $S$. yanoikuyae (Fig. 3), were characterized by having spermidine as the major polyamine in an amended description of the genus, the presence of sym-homospermidine may be a characteristic of the genus Sphingomonas sensu stricto (that is, the species that cluster with $S$. paucimobilis, the type species of the genus).

The substrate utilization profiles and the results of the biochemical investigation demonstrated the separate position of "P. azotocolligans." These results, combined with previously published data, justify a proposal that "P. azotocolligans" should be described as a new species of the genus Sphingomonas. However, the name "P. azotocolligans" did not appear on the Ap- proved Lists of Bacterial Names (19), nor has it been validated. As it has been demonstrated that " $P$. azotocolligans" lacks the ability to fix molecular nitrogen (8), it would be misleading to retain the species epithet. Therefore, we propose the new name Sphingomonas trueperi. The new Sphingomonas species is described below.

Description of Sphingomonas trueperi sp. nov. Sphingomonas trueperi (true'per.i. N. L. gen. n. trueperi, of Trüper, in honor of Hans G. Trüper, a German microbiologist, in recognition of his numerous contributions to the taxonomy of the Proteobacteria) is a gram-negative, rod-shaped, non-spore-forming, motile, peritrichously flagellated organism (5). Colonies are yellow, circular, entire, low convex, and smooth. Growth occurs in the presence of $10 \%$ sodium chloride and $0.02 \%$ sodium azide. Oxidase positive. Catalase positive. Indole, urease, and arginine dihydrolase are not produced. $\beta$-Galactosidase positive. Esculin and gelatin are hydrolyzed. The following compounds are hydrolyzed: $p$-nitrophenyl- $N$-acetyl- $\beta$-D-glucosaminide, $p$ nitrophe-nyl- $\alpha$-L-arabinopyranoside, $p$-nitrophenyl- $\beta$-D-galacuronide, $p$-nitrophenyl- $\alpha$-D-glucopyranoside, $p$-nitrophenyl- $\beta$-Dgluco-pyranoside, $p$-nitrophenyl- $\beta$-D-glucuronide, $p$-nitrophenyl$\beta$-D-melibiose, $p$-nitrophenyl- $\beta$-D-xyloside, bis- $p$-nitrophenylphosphate, $p$-nitrophenyl-phenyl-phosphonate, $p$-nitrophenylphosphoryl-choline, 2-deoxythymidine-5'-p-nitrophenyl-phosphate, D-alanine- $p$-nitroanilide, L-alanine- $p$-nitroanilide, L-arginine- $p$-nitroanilide, $\gamma$-L-glutamate- $p$-nitroanilide, glutamate1- $p$-nitroanilide, glycine- $p$-nitroanilide, L-lysine- $p$-nitroanilide, L-phenylalanine- $p$-nitroanilide, and L-valine- $p$-nitroanilide.

$p$-Nitrophenyl- $N$-acetyl- $\alpha$-D-glucosamine, $p$-nitrophenyl- $\alpha$ - 
TABLE 3. Physiological characteristics of the type strains of 14 Sphingomonas species ${ }^{d}$

\begin{tabular}{|c|c|c|c|c|c|c|c|c|c|c|c|c|c|c|}
\hline Characteristic ${ }^{g}$ & 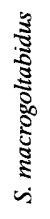 & $\begin{array}{l}\text { पू } \\
\text { हू } \\
\dot{n}\end{array}$ & 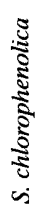 & 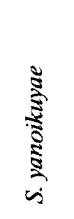 & 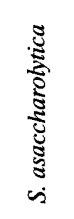 & 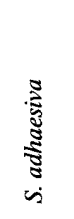 & 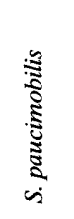 & 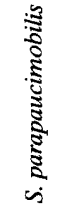 & 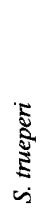 & 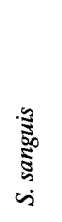 & $\begin{array}{c}\stackrel{\tilde{g}}{2} \\
\stackrel{s}{s}\end{array}$ & 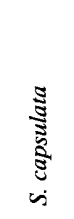 & 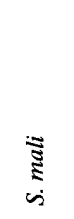 & $\begin{array}{c}\text { है } \\
\text { है } \\
\dot{5}\end{array}$ \\
\hline \multicolumn{15}{|l|}{ Acid produced from ${ }^{e}$ : } \\
\hline Glucose & $-^{f}$ & - & - & $-^{a}$ & $(+)^{c}$ & $-^{a}$ & $-^{a}$ & $-^{a}$ & - & - & $(+)^{c}$ & $-{ }^{a}$ & $(+)^{c}$ & $(+)^{c}$ \\
\hline $\begin{array}{l}\text { Lactose } \\
\text { Sucrose }\end{array}$ & + & - & $\begin{array}{l}- \\
-\end{array}$ & - & $\begin{array}{l}- \\
-\end{array}$ & $\begin{array}{l}- \\
-\end{array}$ & $\begin{array}{l}- \\
-\end{array}$ & $\begin{array}{l}- \\
-\end{array}$ & $\begin{array}{l}- \\
-\end{array}$ & $\begin{array}{l}- \\
-\end{array}$ & $\begin{array}{l}- \\
-\end{array}$ & - & $\begin{array}{l}- \\
-\end{array}$ & - \\
\hline D-Mannitol & - & - & - & $--^{a}$ & + & $-{ }^{a}$ & $-{ }^{a}$ & $-a$ & - & - & - & $-^{a}$ & - & - \\
\hline Dulcitol & + & - & - & $--^{a}$ & - & $-^{a}$ & $--^{a}$ & $-{ }^{a}$ & - & - & - & $-{ }^{a}$ & - & - \\
\hline Salicin & - & - & - & - & $(+)^{c}$ & + & + & - & - & - & $(+)^{c}$ & - & $-^{c}$ & $-^{c}$ \\
\hline Adonitol & - & - & - & $--^{a}$ & - & $-{ }^{a}$ & $--^{a}$ & $-{ }^{a}$ & - & - & - & $-{ }^{a}$ & - & - \\
\hline Inositol & - & + & - & $-a$ & + & $-{ }^{a}$ & $-a$ & $-a$ & - & - & - & $-{ }^{a}$ & - & - \\
\hline Sorbitol & - & - & - & $-a$ & - & $-{ }^{a}$ & $--^{a}$ & $-a$ & + & - & - & $--^{a}$ & - & - \\
\hline L-Arabinose & + & - & - & - & - & - & - & - & - & - & - & - & - & - \\
\hline Raffinose & - & - & - & - & - & - & - & - & - & - & - & - & - & - \\
\hline Rhamnose & - & - & - & $(+)^{a}$ & $(+)^{c}$ & $(+)^{a}$ & $--^{a}$ & $(+)^{a}$ & - & - & $(+)^{c}$ & - & $(+)^{a}$ & $-^{c}$ \\
\hline Maltose & - & - & - & $(+)^{a}$ & - & $(+)^{a}$ & $(+)^{a}$ & $(+)^{a}$ & - & - & - & $(+)^{a}$ & - & - \\
\hline D-Xylose & - & - & + & $(+)^{a}$ & - & $(+)^{a}$ & + & $(+)^{a}$ & - & - & - & $(+)^{a}$ & - & - \\
\hline Trehalose & - & - & - & + & + & - & - & - & - & - & - & - & - & - \\
\hline Cellobiose & - & - & - & $(+)^{a}$ & - & $(+)^{a}$ & $(+)^{a}$ & $(+)^{a}$ & - & - & - & $(+)^{a}$ & - & - \\
\hline Methyl-D-glucoside & - & + & + & - & - & + & + & - & + & - & - & - & - & - \\
\hline Erythritol & - & - & - & - & - & + & - & - & - & - & - & - & - & - \\
\hline Melibiose & - & - & - & $(+)^{a}$ & - & $(+)^{a}$ & $(+)^{a}$ & $(+)^{a}$ & - & - & - & $(+)^{a}$ & - & - \\
\hline D-Arabitol & - & + & - & - & - & - & - & - & - & - & - & - & - & - \\
\hline D-Mannose & - & - & - & $(+)^{a}$ & - & $(+)^{a}$ & $(+)^{a}$ & $(+)^{a}$ & - & - & - & $(+)^{a}$ & - & - \\
\hline \multicolumn{15}{|l|}{ Hydrolysis of: } \\
\hline Esculin & $t^{b}$ & - & - & $+^{a}$ & - & - & $(+)$ & $(+)$ & + & - & $(+)$ & - & $(+)^{r}$ & 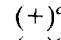 \\
\hline pNP- $\beta$-D-galactopyranoside & + & - & - & $(+)^{a}$ & $(+)^{c}$ & $(+)^{a}$ & $+^{a}$ & $+^{a}$ & + & + & $+^{c}$ & + & $t^{c}$ & $(+)^{c}$ \\
\hline pNP- $\beta$-D-glucuronide & - & - & - & + & + & + & - & + & + & + & + & + & + & + \\
\hline pNP- $\alpha$-D-glucopyranoside & - & + & - & $t^{a}$ & + & $t^{a}$ & $t^{a}$ & $+^{a}$ & + & + & + & $+t^{a}$ & + & + \\
\hline pNP- $\beta$-D-glucopyranoside & + & - & - & $t^{a}$ & + & $t^{a}$ & $t^{a}$ & $+^{a}$ & + & + & + & $+^{a}$ & + & + \\
\hline bis-pNP-phosphate & + & + & + & + & + & + & + & + & + & + & + & + & + & + \\
\hline pNP-phenyl-phosphonate & + & + & + & + & + & + & + & + & + & + & + & + & + & + \\
\hline pNP-phosphoryl-choline & + & + & - & + & + & + & - & + & + & + & + & + & + & + \\
\hline 2-Deoxythymidine-5'-pNP-phosphate & + & + & + & + & + & + & + & + & + & + & + & + & + & + \\
\hline pNP-phosphate & + & + & + & + & + & + & + & + & + & + & + & + & + & + \\
\hline L-Alanine-pNA & + & + & + & + & + & + & + & + & + & + & + & + & + & + \\
\hline L-Glutamate- $\gamma$-3-carboxy-pNA & + & + & + & + & + & + & + & + & + & + & - & + & + & + \\
\hline L-Proline-pNA & + & + & + & - & + & - & - & + & - & - & - & - & + & + \\
\hline \multicolumn{15}{|l|}{ Assimilation of: } \\
\hline$N$-Acetyl-D-glucosamine & - & - & - & $+^{a}$ & $t^{c}$ & $+^{a}$ & $t^{a}$ & $+^{a}$ & + & + & $(+)^{c}$ & $++^{a}$ & + & $t^{c}$ \\
\hline L-Arabinose & - & - & - & $+^{a}$ & $+^{c}$ & $--^{a}$ & $t^{a}$ & $t^{a}$ & + & $t^{b}$ & $+^{c}$ & $(+)^{a}$ & $+^{c}$ & $t^{c}$ \\
\hline$p$-Arbutin & - & - & - & $t^{c}$ & - & + & + & + & + & + & + & + & + & + \\
\hline D-Cellobiose & $-b$ & $-b$ & + & $+^{a}$ & $(+)^{c}$ & $t^{a}$ & $t^{a}$ & $t^{a}$ & + & $t^{b}$ & $t^{c}$ & $t^{a}$ & $t^{c}$ & $+c$ \\
\hline D-Fructose & $-b$ & $-b$ & - & - & - & + & - & - & + & $+^{b}$ & $+^{c}$ & - & - & - \\
\hline D-Galactose & $-b$ & - & - & $t^{a}$ & + & + & + & + & + & $+^{b}$ & - & + & $+^{c}$ & tc \\
\hline Gluconate & $-{ }^{b}$ & $-{ }^{b}$ & - & $+^{c}$ & $-c$ & $-^{c}$ & $-^{c}$ & $+^{c}$ & - & $-{ }^{b}$ & $+^{c}$ & + & $+^{c}$ & $-c$ \\
\hline D-Glucose & + & - & + & $t^{c}$ & $t^{c}$ & + & + & + & + & + & $+^{c}$ & + & $t^{c}$ & $t^{c}$ \\
\hline D-Mannose & $-b$ & $-b$ & - & $-a$ & $t^{c}$ & $t^{a}$ & $t^{a}$ & $t^{a}$ & + & $t^{b}$ & $+c$ & $(+)^{a}$ & $+c$ & $+c$ \\
\hline D-Maltose & - & - & - & $+^{a}$ & $+^{c}$ & $+^{a}$ & $t^{a}$ & $t^{a}$ & + & $t^{b}$ & $+^{c}$ & $+a$ & $+^{c}$ & $t^{c}$ \\
\hline$\alpha$-D-Melibiose & - & - & - & $t^{c}$ & $+^{c}$ & + & - & + & + & + & $t^{c}$ & - & $t^{c}$ & $t^{c}$ \\
\hline L-Rhamnose & - & - & - & $t^{c}$ & $t^{c}$ & - & $-^{c}$ & $(+)^{c}$ & - & - & $t^{c}$ & + & $t^{c}$ & $t^{c}$ \\
\hline D-Ribose & $-b$ & $-b$ & + & $-a$ & - & $-a$ & $-a$ & $-a$ & - & $-b$ & - & $-a$ & - & - \\
\hline Sucrose & - & - & - & $t^{c}$ & $(+)^{c}$ & + & + & + & + & + & $+^{c}$ & + & $+c$ & $t^{c}$ \\
\hline Salicin & - & - & - & $t^{c}$ & $(+)^{c}$ & + & - & + & + & $t^{b}$ & $t^{c}$ & - & $+c$ & $(+)$ \\
\hline D-Trehalose & $t^{b}$ & $-b$ & - & $t^{c}$ & $(+)^{c}$ & + & + & + & + & $t^{b}$ & $-c$ & + & $t^{c}$ & $t^{c}$ \\
\hline D-Xylose & - & - & - & $t^{c}$ & $(+)^{c}$ & $-^{c}$ & $t^{c}$ & $t^{c}$ & + & + & $t^{c}$ & t & $t^{c}$ & $t^{c}$ \\
\hline Adonitol & $-b$ & $-b$ & - & $-{ }^{a}$ & $-c$ & $-{ }^{a}$ & $-^{a}$ & $-^{a}$ & - & $-^{b}$ & $-^{c}$ & $-a$ & $-^{c}$ & $-c$ \\
\hline i-Inositol & - & - & - & $-^{c}$ & $-c$ & - & - & - & - & - & $-^{c}$ & - & $-c$ & $-c$ \\
\hline Maltitol & - & - & - & + & + & - & - & - & + & + & - & - & - & - \\
\hline Putrescine & - & - & - & - & - & - & - & - & - & - & - & - & - & - \\
\hline Acetate & + & - & + & $t^{c}$ & $-c$ & - & - & + & + & + & - & - & + & - \\
\hline Propionate & + & - & + & + & - & - & - & + & + & + & - & - & - & - \\
\hline cis-Aconitate & - & - & - & - & - & - & + & + & - & - & - & - & - & - \\
\hline
\end{tabular}


TABLE 3-Continued

\begin{tabular}{|c|c|c|c|c|c|c|c|c|c|c|c|c|c|c|}
\hline Characteristic $^{g}$ & 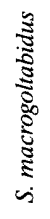 & 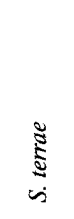 & 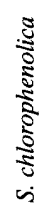 & 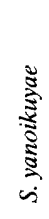 & 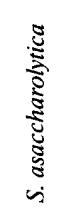 & 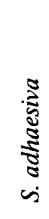 & 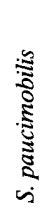 & 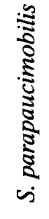 & 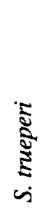 & 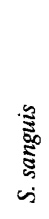 & $\begin{array}{c}\stackrel{g}{0} \\
\stackrel{2}{c} \\
\dot{s}\end{array}$ & 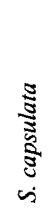 & $\begin{array}{l}\overrightarrow{\bar{g}} \\
\underset{\dot{w}}{\mid}\end{array}$ & 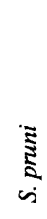 \\
\hline trans-Aconitate & - & - & - & - & - & - & + & - & - & - & - & - & - & - \\
\hline Adipate & $-c$ & $(+)^{c}$ & $-c$ & $-{ }^{a}$ & $--^{c}$ & $-{ }^{a}$ & $-{ }^{a}$ & $-{ }^{a}$ & - & - & $-c$ & $--^{a}$ & $(+)^{c}$ & $-c$ \\
\hline 4-Aminobutyrate & - & - & - & - & - & - & - & - & - & - & - & - & - & - \\
\hline Azelate & + & - & - & - & - & - & - & - & - & - & - & - & - & - \\
\hline Citrate & - & - & - & $t^{c}$ & $--^{c}$ & $-{ }^{c}$ & $-c$ & $+^{c}$ & $--^{c}$ & $-c$ & $-^{c}$ & $-^{c}$ & $-c$ & $-c$ \\
\hline Fumarate & $t^{b}$ & + & + & $t^{c}$ & $(+)^{c}$ & + & + & - & + & + & + & + & $t^{c}$ & $+^{c}$ \\
\hline Glutarate & - & - & - & - & - & - & - & + & + & - & - & - & - & - \\
\hline DL-3-Hydroxybutyrate & + & + & + & + & + & - & - & + & + & + & - & - & + & + \\
\hline Itaconate & - & - & - & - & - & - & - & - & - & - & - & - & - & - \\
\hline DL-Lactate & - & - & - & $t^{c}$ & $--^{c}$ & - & - & + & + & + & $-^{c}$ & - & $-c$ & $-c$ \\
\hline L-Malate & $-b$ & + & + & $t^{c}$ & $--^{c}$ & + & + & + & + & + & + & + & $-c$ & $-c$ \\
\hline Mesaconate & - & - & - & - & - & - & - & - & - & - & - & - & - & - \\
\hline Oxoglutarate & - & - & - & - & - & + & + & + & - & + & - & - & - & - \\
\hline Pyruvate & - & - & + & + & - & - & + & + & + & + & - & - & + & - \\
\hline Suberate & + & - & + & - & - & + & - & - & - & - & + & - & + & - \\
\hline L-Alanine & + & - & - & - & + & + & - & + & + & + & - & - & - & - \\
\hline$\beta$-Alanine & - & - & - & - & - & - & - & - & - & - & - & - & - & - \\
\hline L-Aspartate & + & - & - & + & + & + & - & - & + & + & - & + & + & + \\
\hline L-Histidine & + & - & - & + & - & - & - & - & - & - & + & + & + & + \\
\hline L-Leucine & + & - & + & + & - & + & - & + & + & + & - & + & - & - \\
\hline L-Ornithine & - & - & - & - & - & - & - & - & - & + & - & - & - & - \\
\hline L-Phenylalanine & + & + & - & + & - & - & - & - & - & - & - & - & - & - \\
\hline L-Proline & + & - & - & + & + & + & - & - & - & + & - & - & - & - \\
\hline L-Serine & - & - & - & - & - & - & - & - & + & - & - & - & - & - \\
\hline L-Tryptophan & + & - & - & - & - & - & - & - & - & - & - & - & - & - \\
\hline 3-Hydroxybenzoate & - & - & - & - & - & - & - & - & - & - & - & - & - & - \\
\hline 4-Hydroxybenzoate & - & - & - & + & - & - & - & - & + & - & - & - & - & - \\
\hline Phenylacetate & $-b$ & - & - & $-^{a}$ & - & $-^{a}$ & $-^{a}$ & $-^{a}$ & - & $-^{b}$ & - & $-^{a}$ & - & - \\
\hline
\end{tabular}

${ }^{a}$ Data are in agreement with the data of Yabuuchi et al. (22).

${ }^{b}$ Data are in agreement with the data of Takeuchi et al. (18)

- Data are in agreement with the data of Takeuchi et al. (19).

${ }^{d}$ Test results were determined after $72 \mathrm{~h}$ of incubation at $30^{\circ} \mathrm{C}$.

' Acid formation from carbohydrates in most cases was very weak (even after prolonged incubation); these tests cannot be recommended for differentiation.

$f$-, negative; + , positive; $(+)$, weakly positive.

${ }^{g}$ pNP, para-nitrohenyl; pNA, para-nitroanilide.

L-fucopyranoside, $p$-nitrophenyl- $\beta$-D-galactosaminide, $p$-nitro-phenyl- $\beta$-D-galactopyranoside, $p$-nitrophenyl- $\beta$-D-cellobioside, $p$-nitrophenyl- $\beta$-D-fucopyranoside, $p$-nitrophenyl- $\beta$-Dlacto-side, $p$-nitrophenyl- $\alpha$-D-maltoside, $p$-nitrophenyl- $\beta$-Dmaltoside, $p$-nitrophenyl- $\alpha$-D-mannopyranoside, $p$-nitro phenyl- $\alpha$-L-rhamnopyranoside, $p$-nitrophenyl-sulfate, acetyl-Lalanine- $p$-nitro-anilide, L-glutamate- $\gamma$-3-carboxyl-p-nitroanilide, succinyl-L-phenylalanine- $p$-nitroanilide, acetyl-L-leucine$p$-nitroanilide, benzoyl-DL-arginine- $p$-nitroanilide, and Lproline- $p$-nitroani-lide are not hydrolyzed.

The following compounds are assimilated: D-glucose, L-arabinose, D-mannose, D-xylose, D-fructose, $\alpha$-methyl-D-mannoside, $\alpha$-methyl-D-glucoside, esculin, salicin, lactose, sucrose, trehalose, D-raffinose, amidon, $\beta$-gentiobiose, D-turanose, Lfucose, L-malate, DL-malate, succinate, fumarate, DL-lactate, DL-3-hydroxybutyrate, pyruvate, $N$-acetyl-D-glucosamine, $p$-arbutin, D-cellobiose, D-galactose, D-glucosamine, D-glucuronate, lactulose, D-maltose, D-mannoheptulose, $\alpha$-D-melezitose, $\alpha$-Dmelibiose, $\alpha$-methyl-D-mannoside, $\alpha$-methyl-D-glucopyranoside, starch, D-sucrose, D-trehalose, acetamidocaprate, acetyl-L-glutamine, acetyl-L-proline, L-alanine, DL-2-aminoadipate, L-asparagine, L-aspartate, DL-aspartate, D-glutamate, L-glutamate, L-glutamine, L-isoleucine, L-leucine, phenylglycine, L-proline,

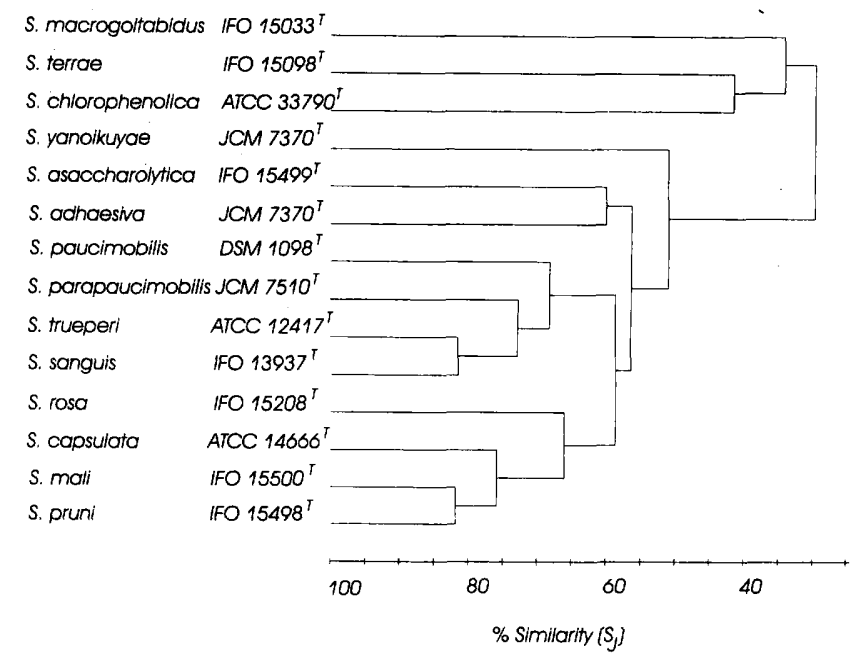

FIG. 2. Phenogram of Sphingomonas species generated by using the UPGMA algorithm and $S_{J}(20)$. 


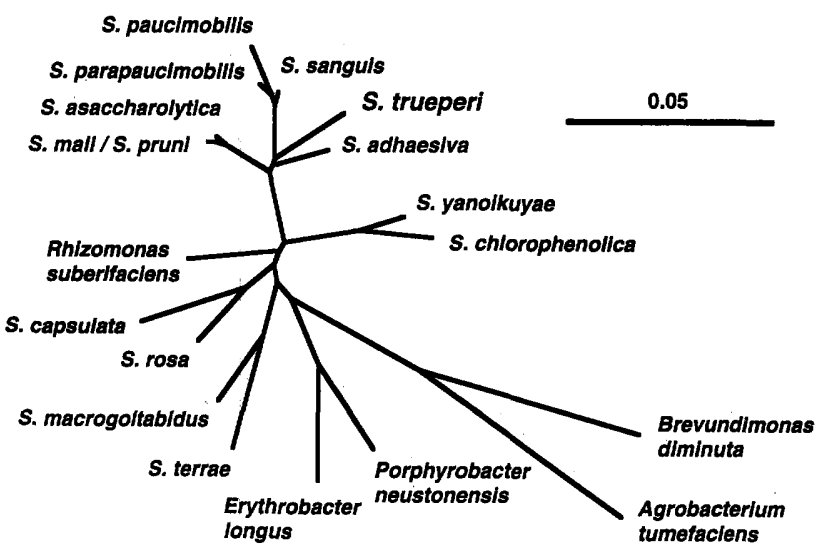

FIG. 3. Unrooted dendrogram showing the estimated phylogenetic relationships between $S$. trueperi and other species of the genus Sphingomonas as derived by evolutionary distance calculations from pairwise comparisons of nearly complete 16S rRNA gene sequences, including the Jukes-Cantor correction factor. Nucleotide insertions-deletions were weighted 0.5 times the value of nucleotide substitutions. The dendrogram was generated by using the least-squares distance algorithm of the FITCH program in PHYLIP (Phylogeny Inference Package, version $3.5 \mathrm{c}$ ). The EMBL accession numbers for sequences obtained from the EMBL Nucleotide Sequence Database are as follows: $S$. paucimobilis DSM $1098^{\mathrm{T}}, \mathrm{X} 72722 ;$ S. adhaesiva JCM $7370^{\mathrm{T}}, \mathrm{X} 72720 ;$ S. asaccharolytica IFO $15499^{\mathrm{T}}$ Y09639; S. capsulata IFO 12533 ${ }^{\mathrm{T}}$, D16147; S. chlorophenolica ATCC $33790^{\mathrm{T}}$ $\mathrm{X} 87161 ; S$. macrogoltabidus IFO $15033^{\mathrm{T}}$, D13723; $S$. mali IFO $15500^{\mathrm{T}}$, Y09638 $S$, parapaucimobilis JCM $7510^{\mathrm{T}}$ X72721; $S$. pruni IFO $15498^{\mathrm{T}}$, Y09637; S. rosa IFO $15208^{\mathrm{T}}$ D13945; $S$. sanguis IFO $13937^{\mathrm{T}}$, D13726; $S$. terrae IFO $15098^{\mathrm{T}}$ D13727; S. yanoikuyae JCM $7371^{\mathrm{T}}, \mathrm{X} 72725 ;$ Rhizomonas suberifaciens IFO 15211 ${ }^{\mathrm{T}}$, D13737; Agrobacterium tumefaciens DSM 30150, M11223; Brevundimonas diminuta ATCC $11568^{\mathrm{T}}$, M59064; Erythrobacter longus JCM $6170^{\mathrm{T}}$, D12699 and Porphyrobacter neustonensis ACM $2844^{\mathrm{T}}$, L01785. Bar $=5$ substitutions per 100 nucleotide positions (estimated evolutionary distance from the point of divergence of the $16 \mathrm{~S}$ rRNA gene sequences)

L-valine, casein, malitol, tributyrin, DL-2-hydroxyisobutyrate, 2hydroxyvalerate, oxalacetate, 2-oxoglutarate, 2-oxoisocaprate, 1-butanol, L-leucineamide, $p$-hydroxybenzoate, and protocatechuate.

Acid is produced from $N$-acetyl-D-glucosamine, L-arabinose, D-cellobiose, D-fructose, D-galactose, D-glucosamine, D-glucose, lactose, lactulose, D-mannose, salicin, D-trehalose, Dturanose, and D-xylose.

The following compounds are not assimilated: glycerol, erythritol, D-arabinose, ribose, L-xylose, adonitol, $\beta$-methyl-xyloside, L-sorbose, rhamnose, dulcitol, sorbitol, inulin, glycogen, L-lyxose, D-tagatose, D-fucose, D-arabitol, L-arabitol, 5-ketogluconate, $N$-acetylgalactosamine, amygdalin, $\alpha$-D-galacturonate, D-glucarate, D-glucosaminic acid, glycogen, glycyrrhizinate, L-mannose, $\beta$-methyl-D-xyloside, mucate, 5-oxogluconate, L-rhamnose, D-ribose, $m$-erythritol, i-inositol, D-mannitol, D-xylitol, 1-hexanol, acetyl-L-glutamate, acetylglycine, acetyl-DL-methionine, D-alanine, 4-aminobutyrate, $\mathrm{L}$-arginine, betaine, L-citrulline, dimethylglycine, L-histidine, L-hydroxyproline, DL-isoleucine, D-leucine, L-lysine, D-norvaline, L-norvaline, L-ornithine, L-phenylalanine, D-proline, sarcosine, L-serine, L-threonine, carnitine, creatine, putrescine, spermidine, tyramine, allantoine, cytosine, glycinamide, acetate, propionate, butyrate, isobutyrate, $n$-valerate, isovalerate, $N$-caproate, heptanoate, caprylate, pelargonate, caprate, oxalate, malonate, maleate, glutarate, adipate, pimelate, suberate, azelate, sebacate, cis-aconitate, transaconitate, glycerophosphate, glyoxylate, 4-hydroxybutyrate, DL2-hydroxyisovalerate, D-lactate, L-lactate, mesoxalate, 2-butanol, ethanol, hippurate, thiamine, ureidosuccinate, L-valinamide, anthranilate, 3-aminobenzoate, 4-aminobenzoate, 3-hydroxyphenylacetate, 4-hydroxyphenylacetate, quinate, salicylate, tropic acid, vanillate, glycolate, DL-glycerate, $\mathrm{D}$-malate, $\mathrm{D}$-tartrate, $\mathrm{L}$ - tartrate, $m$-tartrate, levulinate, 2-ketogluconate, citraconate, itaconate, mesaconate, aconitate, citrate, phenylacetate, benzoate, $o$-hydroxybenzoate, $m$-hydroxybenzoate, D-mandelate, L-mandelate, phthalate, isophthalate, terephthalate, arabonic acid, dextran, sorbate, L-2-hydroxyisocaprate, DL-isocitrate, tartronate, 1,8-octandiol, $\beta$-alanine, DL-aminobutyrate, DL3-aminobutyrate, DL-2-aminoisobutyrate, aminoxyacetate, Darginine, D-asparagine, L-cysteinate, DL-2,4-diaminobutyrate, diaminopimelate, 2,3-diaminopropionate, L-glycine, glycinanhydride, D-histidine, L-homoserine, DL- $\delta$-hydroxylysine, D-lysine, D-methioninesulfone, L-norleucine, D-phenylalanine, serine, D-tryptophan, L-tryptophan, butylamine, cadaverine, ethanolamine, ethylamine, urea, hexylamine, spermine, taurine, trigonelline, tryptamine, acetamide, indole-3-acetate, oxamide acid, salicylamide, uracil, furan-2-carbonic acid, 2-hydroxyphenylacetate, phenoxyacetate, phenyllactate, phenylpropionate, and phenylpyruvate. Acetate, butyrate, isobutyrate, isovalerate, malonate, glutarate, glycerate, glycolate, and propionate are weakly assimilated after 7 days of incubation.

No acid is produced from D-arabinose, arabonic acid, dextran, $\alpha$-D-galacturonate, gluconate, glucuronate, inulin, lactobionic acid, L-lyxose, D-maltose, glycogen, $\alpha$-D-melezitose, $\alpha$-D-melibiose, L-rhamnose, D-sucrose, $\alpha$-methyl-D-mannoside, methyl- $\alpha$-D-glucopyranoside, $\beta$-methyl-D-xyloside, mucate, $\alpha$ D-raffinose, D-ribose, L-sorbose, starch, D-tagatose, L-xylose, dulcitol, $m$-erythritol, glycerol, i-inositol, maltitol, D-xylitol, and tributyrin.

The $\mathrm{G}+\mathrm{C}$ content of the DNA is $65.6 \mathrm{~mol} \%$ (5). The major isoprenoid quinone is ubiquinone Q-10. The main component in the polyamine pattern is sym-homospermidine (4). The major nonpolar fatty acids are 18:1 and 16:0, and the major 2-hydroxy fatty acid is 14:0 $2 \mathrm{OH}$.

Sphingoglycolipid, phosphatidylethanolamine, phosphatidylglycerol, diphosphatidylglycerol, phosphatidyldimethylethanolamine, and an unknown lipid are the predominant polar lipids. Phosphatidylmonomethylethanolamine and four unknown lipids are present in minor concentrations.

Isolated from soil.

TABLE 4. Levels of sequence similarity between the 16S rRNA gene of $S$. trueperi and the 16S rRNA genes of other Sphingomonas species and species belonging to the $\alpha$ subclass of the Proteobacteria

\begin{tabular}{|c|c|c|}
\hline Species & Strain $^{a}$ & $\begin{array}{l}\text { \% Sequence similar- } \\
\text { ity to } S \text {. trueperi }\end{array}$ \\
\hline Sphingomonas paucimobilis & DSM $1098^{\mathrm{T}}$ & 95.8 \\
\hline Sphingomonas parapaucimobilis & JCM $7510^{\mathrm{T}}$ & 95.4 \\
\hline Sphingomonas adhaesiva & $\mathrm{JCM} 7370^{\mathrm{T}}$ & 95.5 \\
\hline Sphingomonas sanguis & IFO $13937^{\mathrm{T}}$ & 95.6 \\
\hline Sphingomonas mali & IFO $15500^{\mathrm{T}}$ & 95.8 \\
\hline Sphingomonas pruni & IFO $15498^{\mathrm{T}}$ & 95.8 \\
\hline Sphingomonas asaccharolytica & IFO $15499^{\mathrm{T}}$ & 95.8 \\
\hline Sphingomonas yanoikuyae & $\mathrm{JCM} 7370^{\mathrm{T}}$ & 93.1 \\
\hline Sphingomonas chlorophenolica & $\operatorname{ATCC} 33790^{\mathrm{T}}$ & 92.4 \\
\hline Sphingomonas macrogoltabidus & IFO $15033^{\mathrm{T}}$ & 91.1 \\
\hline Sphingomonas terrae & IFO $15098^{\mathrm{T}}$ & 90.9 \\
\hline Sphingomonas capsulata & ATCC $14666^{\mathrm{T}}$ & 90.8 \\
\hline Sphingomonas rosa & IFO $15208^{\mathrm{T}}$ & 92.1 \\
\hline Rhizomonas suberifaciens & IFO $15211^{\mathrm{T}}$ & 92.4 \\
\hline Enythrobacter longus & $\mathrm{JCM} 6170^{\mathrm{T}}$ & 90.9 \\
\hline Brevundimonas diminuta & ATCC $11568^{\mathrm{T}}$ & 87.2 \\
\hline Agrobacterium tumefaciens & DSM 30150 & 86.4 \\
\hline
\end{tabular}

${ }^{a}$ DSM, Deutsche Sammlung von Mikroorganismen und Zellkulturen, Braunschweig, Germany; JCM, Japanese Collection of Microorganisms, Wako, Saitama, Japan; IFO, Institute of Fermentation, Yodogawa-ku, Osaka, Japan ATCC, American Type Culture Collection, Rockville, Md. 
The type strain is strain NCIMB 9391 (= ATCC $12417=$ LMG 2142 = DSM 7225).

The 16S rDNA sequence has been deposited in the EMBL data bank under accession number X 97776 .

H.-J.B. acknowledges Lise Meitner fellowship M00159-MOB from the Austrian Science Foundation. E.R.B.M. was supported in part by the Commission of the European Communities under contracts BIOTCT91-0294 and BIO2-CT94-3098.

\section{REFERENCES}

1. American Type Culture Collection. 1989. Catalogue of strains, 17th ed. American Type Culture Collection, Rockville, Md.

1a.Anderson, G. R. 1955. Nitrogen fixation by Pseudomonas-like soil bacteria J. Bacteriol. 70:129-133.

2. Auling, G., H.-J. Busse, F. Pilz, L. Webb, H. Kneifel, and D. Claus. 1991 Rapid differentiation by polyamine analysis of Xanthomonas strains from phytopathogenic pseudomonads and other members of the class Proteobacteria interacting with plants. Int. J. Syst. Bacteriol. 41:223-228.

3. Bauwens, M., and J. De Ley. 1981. Improvements in the taxonomy of Fla vobacterium by DNA:rRNA hybridization, p. 27-31. In $\mathrm{H}$. Reichenbach and O. B. Weeks (ed.), The Flavobacterium-Cytophaga group. Verlag Chemie, Weinheim, Germany.

4. Busse, J., and G. Auling. 1988. Polyamine pattern as a chemotaxonomic marker within the Proteobacteria. Syst. Appl. Microbiol. 11:1-8

5. De Ley, J. 1968. DNA base composition and classification of some more free-living nitrogen-fixing bacteria. Antonie van Leeuwenhoek 34:66-70.

6. De Vos, P., and J. De Ley. 1983. Intra- and intergeneric similarities of Pseudomonas and Xanthomonas ribosomal ribonucleic acid cistrons. Int. J. Syst. Bacteriol. 33:487-509.

7. De Vos, P., A. Van Landschoot, P. Segers, R. Tytgat, M. Gillis, M. Bauwens, R. Rossau, M. Goor, B. Pot, K. Kersters, P. Lizzaraga, and J. De Ley. 1989. Genotypic relationships and taxonomic localization of unclassified Pseudomonas and Pseudomonas-like strains by deoxyribonucleic acid-ribosomal ribonucleic acid hybridizations. Int. J. Syst. Bacteriol. 39:35-49.

8. Hill, S., and J. R. Postgate. 1969. Failure of putative nitrogen-fixing bacteria to fix nitrogen. J. Gen. Microbiol. 58:277-285.

9. Kämpfer, P., and M. Altwegg. 1992. Numerical classification and identification of Aeromonas genospecies. J. Appl. Bacteriol. 72:341-351.

10. Kämpfer, P., K. Bark, H.-J. Busse, G. Auling, and W. Dott. 1992. Numerical and chemotaxonomy of polyphosphate accumulating Acinetobacter strains with high polyphosphate:AMP phosphotransferase (PPAT) activity. Syst. Appl. Microbiol. 15:309-419.

11. Kämpfer, P., and R. M. Kroppenstedt. 1996. Numerical analysis of fatty acid patterns of coryneform bacteria and related taxa. Can. J. Microbiol. 42: 989-1005.

12. Kämpfer, P., M. Steiof, and W. Dott. 1991. Microbiological characterization of a fuel-oil contaminated site including numerical identification of hetero- trophic water and soil bacteria. Microb. Ecol. 21:227-251.

13. Karlson, U., F. Rojo, J. D. van Elsas, and E. Moore. 1996. Genetic and serological evidence for the recognition of four pentachlorophenol-degrad ing bacterial strains as a species of the genus Sphingomonas. Syst. Appl. Microbiol. 18:539-548.

14. Kroppenstedt, R. M. 1982. Anwendung chromatographischer HP-Verfahren (HPTLC und HPLC) in der Bakterientaxonomie. GIT Lab. Med. 5:266-275.

15. Moore, E. R. B., R.-M. Wittich, P. Fortnagel, and K. N. Timmis. 1993. 16 S ribosomal RNA gene sequence characterization and phylogenetic analysis of a dibenzo-p-dioxin-degrading isolate within the new genus Sphingomonas. Lett. Appl. Microbiol. 17:115-118.

16. Nohynek, L., E. Suhonen, E.-L. Nurmiaho-Lassila, J. Hantula, and M. Salkinoja-Salonen. 1996. Description of four pentachlorophenol-degrading bacterial strains as Sphingomonas chlorophenolica sp. nov. Syst. Appl. Microbiol. 18:527-538.

17. Oyaizu, H., and K. Komagata. 1983. Grouping of Pseudomonas species on the basis of cellular fatty acid composition and the quinone system with special reference to the existence of 3-hydroxy fatty acids. J. Gen. Appl. Microbiol. 29:17-40.

18. Segers, P., M. Vancanneyt, B. Pot, U. Torck, B. Hoste, D. Dewettinck, E Falsen, K. Kersters, and P. De Vos. 1994. Classification of Pseudomonas diminuta Leifson and Hugh 1954 and Pseudomonas vesicularis Büssing, Döll, and Freytag 1953 in Brevundimonas gen. nov, and Brevundimonas vesiculari comb. nov., respectively. Int. J. Syst. Bacteriol. 44:499-510.

19. Skerman, V. B. D., V. McGowan, and P. H. A. Sneath (ed.). 1980. Approved lists of bacterial names. Int. J. Syst. Bacteriol. 30:225-420.

20. Sneath, P. H. A., and R. R. Sokal. 1973. Numerical taxonomy. The principles and practice of numerical classification. W. H. Freeman, San Francisco Calif.

21. Takeuchi, M., F. Kawai, Y. Shimada, and A. Yokota. 1993. Taxonomic study of polyethylene glycol-utilizing bacteria: amended description of the genus Sphingomonas and new description of Sphingomonas macrogoltabidus sp. nov., Sphingomonas sanguis sp. nov., and Sphingomonas terrae sp. nov. Syst. Appl. Microbiol. 16:227-238.

22. Takeuchi, M., T. Sakane, M. Yanagi, K. Yamasato, K. Hamana, and A. Yokota. 1995. Taxonomic study of bacteria isolated from plants: proposal of Sphingomonas rosa sp. nov., Sphingomonas pruni sp. nov., Sphingomona. asaccharolytica sp. nov., and Sphingomonas mali sp. nov. Int. J. Syst. Bacteriol. 45:334-341.

23. Tindall, B. J. 1990. Lipid composition of Halobacterium lacusprofundi. FEMS Microbiol. Lett. 66:199-202.

24. Woese, C. R., P. Blanz, and C. M. Hahn. 1984. What isn't a pseudomonad: the importance of nomenclature in bacterial classification. Syst. Appl. Microbiol. 5:179-195.

25. Yabuuchi, E., I. Yano, H. Oyaizu, Y. Hashimoto, T. Ezaki, and H. Yamamoto. 1990. Proposals of Sphingomonas paucimobilis gen. nov. and comb. nov., Sphingomonas parapaucimobilis sp. nov., Sphingomonas yanoikuyae sp. nov., Sphingomonas adhaesiva sp. nov., Sphingomonas capsulata comb. nov., and two genospecies of the genus Sphingomonas. Microbiol. Immunol. 34: 99-119. 